\title{
Multiple positive solutions for perturbed nonlinear fractional differential system with two control parameters
}

\author{
Yulin Zhao', Xiaoyan Shi ${ }^{1 *}$ and Liang Tang ${ }^{1}$
}

\section{"Correspondence:}

xiaoyshi08@126.com

'School of Science, Hunan

University of Technology, Zhuzhou,

P.R. China

\begin{abstract}
In this paper, we study a class of perturbed nonlinear fractional differential systems with two control parameters. Under some appropriate conditions on the primitive function of nonlinear terms $F_{u}$ and $F_{v}$, we prove the multiplicity of weak solutions by using the variational method and Ricceri's critical points theorems.
\end{abstract}

MSC: Primary 34A08; secondary 34B37; 58E05

Keywords: Three solutions; Perturbed fractional differential system; Variational method; Critical points

\section{Introduction}

It is commonly known that fractional differential equations (FDEs) are a generalization of ordinary differential equations and integration of arbitrary (noninteger) orders. With the help of fractional calculus, the natural phenomena and mathematical models in different fields of science and engineering can be accurately described. FDEs have also plentiful applications in such fields as chemistry, electrochemistry, biology, mechanics, polymer rheology, economics, control theory, viscoelasticity and damping, blood flow phenomena, biophysics, and so on (see $[1,2]$ and the references therein). Much important advances have also been made in the theory of fractional calculus and fractional ordinary and partial differential equations; for more detail on fractional calculus theory and applications, we refer the reader to the monographs of Podlubny [3], Diethelm [1], Kilbas et al. [2], and Zhou [4].

Applying various tools and techniques of nonlinear analysis as the fixed point theorems, the coincidence degree theory, the monotone iterative methods, critical point theory, and variational methods, many researchers have explored the existence and multiplicity of solutions for nonlinear fractional initial and boundary value problems (see [5-28] and the references therein), and coupled systems of nonlinear FDEs; see, for ecample, [13, 18, 19, 29-32]. Ahmad and Alsaedi [13] studied the following fractional differential system:

$$
\left\{\begin{array}{lll}
{ }^{\mathrm{c}} D_{T}^{\rho} u(t)=f\left(t,{ }^{\mathrm{c}} D^{\alpha} v(t)\right), & u^{(k)}=\eta_{k}, & 0<t<1, \\
{ }^{\mathrm{c}} D_{T}^{\sigma} v(t)=g\left(t,{ }^{\mathrm{c}} D^{\beta} u(t)\right), & u^{(k)}=\xi_{k}, & 0<t<1,
\end{array}\right.
$$

(c) The Author(s) 2019. This article is distributed under the terms of the Creative Commons Attribution 4.0 International License (http://creativecommons.org/licenses/by/4.0/), which permits unrestricted use, distribution, and reproduction in any medium, provided you give appropriate credit to the original author(s) and the source, provide a link to the Creative Commons license, and indicate if changes were made. 
where ${ }^{\mathrm{c}} D$ denotes the Caputo fractional derivative, $\rho, \sigma \in(m-1, m), \alpha, \beta \in(n-1, n), m, n \in$ $\mathbf{N}, \rho>\alpha, \sigma>\beta, k=0,1,2, \ldots, m-1, \rho, \sigma, \alpha, \beta \notin \mathbf{N}$, and $\eta_{k}, \xi_{k}$ are suitable real constants. By applying the nonlinear alternative of Leray-Schauder theorem in a cone the existence and uniqueness results for such coupled systems are obtained.

Based on variational methods and critical point theory, Jiao and Zhou [21] investigated the existence of a weak solution to the following nonlinear FDEs:

$$
\left\{\begin{array}{l}
{ }_{t} D_{T}^{\alpha}\left({ }_{0} D_{t}^{\alpha} u(t)\right)=\nabla F(t, u(t)), \quad \text { a.e. } t \in[0, T], \\
u(0)=u(T)=0
\end{array}\right.
$$

where $\alpha \in(0,1],{ }_{0} D_{t}^{\alpha}$ and ${ }_{t} D_{T}^{\alpha}$ are the left and right Riemann-Liouville fractional derivatives, respectively. $F:[0, T] \times \mathbf{R}^{\mathbf{N}} \rightarrow \mathbf{R}(N \geq 1)$ is an appropriate given function, and $\nabla F(t, u)$ is the gradient of $F$ at $u$.

Galewski and Molica Bisci [26] considered the following one-dimensional fractional problem:

$$
\left\{\begin{array}{l}
\frac{d}{d t}\left({ }_{0} D_{t}^{\alpha-1}\left({ }_{0}^{\mathrm{c}} D_{t}^{\alpha} u(t)\right)-{ }_{t} D_{T}^{\alpha-1}\left({ }_{t}^{\mathrm{c}} D_{T}^{\alpha} u(t)\right)\right)+f(t, u(t))=0, \quad \text { a.e. } t \in[0, T], \\
u(0)=u(T)=0,
\end{array}\right.
$$

where $\alpha \in\left(\frac{1}{2}, 1\right],{ }^{\mathrm{c}} D$ denotes the Caputo fractional derivative, ${ }_{0} D_{t}^{\alpha-1}$ and ${ }_{t} D_{T}^{\alpha-1}$ are the left and right Riemann-Liouville fractional derivatives of order $\alpha-1$, respectively. By using variational methods the existence of at least one nontrivial solution for this onedimensional fractional problem has been obtained under an asymptotical behavior of the nonlinear term at zero.

Bai [15] studied the multiplicity of weak solutions for the following perturbed nonlinear FDEs:

$$
\left\{\begin{array}{l}
{ }_{t} D_{T}^{\alpha}\left({ }_{0} D_{t}^{\alpha} u(t)\right)=\lambda a(t) f(u(t))+\mu g(t, u(t)), \quad \text { a.e. } t \in[0, T], \\
u(0)=u(T)=0,
\end{array}\right.
$$

where $\alpha \in(0,1],{ }_{0} D_{t}^{\alpha}$ and ${ }_{t} D_{T}^{\alpha}$ are the left and right Riemann-Liouville fractional derivatives, respectively, $\lambda, \mu$ are nonnegative parameters, and $f: \mathbf{R} \rightarrow \mathbf{R}, g:[0, T] \times \mathbf{R} \rightarrow \mathbf{R}$, and $a:[0, T] \rightarrow \mathbf{R}$ are continuous functions. By using a recent variational principle of Bonanno and Molica Bisci [33], some sufficient criteria of the existence of infinitely many solutions depending on the parameters $\lambda$ and $\mu$ are established.

Zhao et al. [31] studied the existence of weak solutions for the following coupled nonlinear fractional differential system:

$$
\begin{cases}{ }_{t} D_{T}^{\alpha}\left(a(t){ }_{0} D_{t}^{\alpha} u(t)\right)=\lambda F_{u}(t, u(t), v(t)), & 0<t<T, \\ { }_{t} D_{T}^{\beta}\left(b(t){ }_{0} D_{t}^{\beta} v(t)\right)=\lambda F_{v}(t, u(t), v(t)), & 0<t<T, \\ u(0)=u(T)=0, \quad v(0)=v(T)=0, & \end{cases}
$$

where $\alpha, \beta \in(0,1], \lambda$ is a nonnegative real parameter, $F_{u}$ and $F_{v}$ denote the partial derivatives of $F$ with respect to $u, v$, respectively, and $F:[0, T] \times \mathbf{R}^{2} \rightarrow \mathbf{R}$ is a suitable given function such that $F(\cdot, x, y)$ is continuous in $[0, T]$ for any $(x, y) \in \mathbf{R}^{2}$. By using a potent method 
due to Bonanno and Marano [34], some sufficient conditions for the existence of at least three weak solutions on the parameter $\lambda$ are obtained. For applications and examples of fractional-order systems, we refer the reader to [35-38].

Motivated by the papers mentioned, in this paper, we are interested in the existence results for the following perturbed fractional differential system:

$$
\left\{\begin{array}{l}
{ }_{t} D_{T}^{\alpha}\left(a(t)_{0} D_{t}^{\alpha} u(t)\right)=\lambda F_{u}(t, u, v)+\mu G_{u}(t, u, v)+h_{1}(u), \quad 0<t<T, \\
{ }_{t} D_{T}^{\beta}\left(b(t){ }_{0} D_{t}^{\beta} v(t)\right)=\lambda F_{v}(t, u, v)+\mu G_{v}(t, u, v)+h_{2}(v), \quad 0<t<T, \quad\left(P_{\lambda, \mu}\right) \\
u(0)=u(T)=0, \quad v(0)=v(T)=0,
\end{array}\right.
$$

where $\lambda, \mu$ are positive real parameters, $0<\alpha, \beta \leq 1, a, b \in L^{\infty}[0, T]$ with $a_{0}:=$ essinf ${ }_{[0, T]} a(t)>0$ and $b_{0}:=\operatorname{essinf}_{[0, T]} b(t)>0,{ }_{0} D_{t}^{\gamma}$ and ${ }_{t} D_{T}^{\gamma}$ denote the left and right Riemann-Liouville fractional derivatives of order $\gamma$, respectively. For convenience, we list the following assumptions on $F, G$ and $h_{1}, h_{2}$.

(F0) $F:[0, T] \times \mathbf{R}^{2} \rightarrow \mathbf{R}$ is a function such that $F(\cdot, u, v)$ is continuous in $[0, T]$ for any $(u, v) \in \mathbf{R}^{2}, F(t, \cdot, \cdot)$ is a $C^{1}$ function in $\mathbf{R}^{2}$, and $F_{s}$ is the partial derivative of $F$ with respect to $s$;

(G0) $G:[0, T] \times \mathbf{R}^{2} \rightarrow \mathbf{R}$ is measurable with respect to $t$ for every $(u, v) \in \mathbf{R}^{2}$, continuously differentiable in $\mathbf{R}^{2}$ for a.e. $t \in[0, T]$, and $G_{u}, G_{v}$ denote the partial derivatives of $G$ that satisfy the following condition:

$$
\left.\sup _{\sqrt{u^{2}+v^{2}} \leq \xi} \max \left\{\left|G_{u}(\cdot, u, v)\right|,\left|G_{v}(\cdot, u, v)\right|\right)\right\} \in L^{1}([0, T]) \quad \text { for all } \xi>0
$$

(H0) $h_{1}, h_{2}: \mathbf{R} \rightarrow \mathbf{R}$ are Lipschitz continuous functions satisfying $h_{i}(0)=0, i=1,2$, with Lipschitz constants $L_{1}, L_{2} \geq 0$, that is,

$$
\left|h_{i}\left(x_{1}\right)-h_{i}\left(x_{2}\right)\right| \leq L_{i}\left|x_{1}-x_{2}\right|, \quad i=1,2,
$$

for all $x_{1}, x_{2} \in \mathbf{R}$.

In this paper, by defining an appropriate functional space and constructing the corresponding variational framework, we employ a potent method due to Ricceri [39-41] to deal with the system $\left(P_{\lambda, \mu}\right)$ and gain several new existence results for weak solutions in terms of distinct values of the parameters $\lambda, \mu$ belonging to real intervals. It is worth remarking that we obtain the multiplicity results for two cases: where the primitive function $F$ of $F_{u}$ and $F_{v}$ is subquadratic and where it is asymptotically quadratic as $|(u, v)| \rightarrow \infty$. In addition, we assume that the primitive function $G$ of $G_{u}$ and $G_{v}$ satisfies a general growth condition allowing us to apply the variational method. We present two examples to illustrate the applicability of our main results.

\section{Preliminaries and variational formulation}

To apply critical point theory to investigate the existence of weak solutions for the system $\left(P_{\lambda, \mu}\right)$, we recall some basic notations and lemmas and construct a variational framework.

Let $X$ be a real Banach space, and let $\Upsilon_{X}$ denote the class of all functionals $\Phi: X \rightarrow \mathbf{R}$ that possess the following property: if $\left\{\omega_{n}\right\}$ is a sequence in $X$ converging weakly to $\omega \in X$ and $\lim _{n \rightarrow \infty} \inf \Phi\left(\omega_{n}\right) \leq \Phi(\omega)$, then $\left\{\omega_{n}\right\}$ admits a subsequence converging strongly to $\omega$. For 
example, if $X$ is uniformly convex and $\phi:[0,+\infty) \rightarrow \mathbf{R}$ is a continuous strictly increasing function, then the functional $\omega \rightarrow \phi(\|\omega\|)$ belongs to the class $\Upsilon_{X}$.

Theorem 2.1 (see [39]) Let $X$ be a separable reflexive real Banach space, and let $\Phi$ : $X \rightarrow \mathbf{R}$ be a coercive sequentially weakly lower semicontinuous, $C^{1}$ functional belonging to $\Upsilon_{X}$, bounded on each bounded subset of $X$, with derivative admitting a continuous inverse on $X^{*}$. Let $\Psi: X \rightarrow \mathbf{R}$ be a $C^{1}$ functional with compact derivative. Assume that $\Phi$ has a strict local minimum $x_{0}$ with $\Phi\left(x_{0}\right)=\Psi\left(x_{0}\right)=0$. Finally, setting

$$
\begin{aligned}
& \delta_{1}=\max \left\{0, \limsup _{\|x\| \rightarrow+\infty} \frac{\Psi(x)}{\Phi(x)}, \limsup _{x \rightarrow x_{0}} \frac{\Psi(x)}{\Phi(x)}\right\}, \\
& \delta_{2}=\sup _{x \in \Phi^{-1}((0,+\infty))} \frac{\Psi(x)}{\Phi(x)},
\end{aligned}
$$

we assume that $\delta_{1}<\delta_{2}$.

Then, for each compact interval $[a, b] \subset\left(\frac{1}{\delta_{2}}, \frac{1}{\delta_{1}}\right)$ (with the conventions $\frac{1}{0}=+\infty$ and $\frac{1}{+\infty}=$ $0)$, there exists $\varrho>0$ with the following property: for every $\lambda \in[a, b]$ and every $C^{1}$ functional $J: X \rightarrow \mathbf{R}$ with compact derivative, there exists $\mu^{*}>0$ such that, for each $\mu \in\left[0, \mu^{*}\right]$, the equation

$$
\Phi^{\prime}(x)=\lambda \Psi^{\prime}(x)+\mu J^{\prime}(x)
$$

has at least three solutions in $X$ with norms less than $\varrho$.

We need the following two results of Ricceri to guarantee the existence of three solutions for a given equation.

Theorem 2.2 (see [40]) Let $X$ be a reflexive real Banach space, and let $I \subset \mathbf{R}$ be an interval. Let $\Phi: X \rightarrow \mathbf{R}$ be a sequentially weakly lower semicontinuous, $C^{1}$ functional bounded on each bounded subset of $X$, with derivative admitting a continuous inverse on $X^{*}$. Let $-\Psi$ : $X \rightarrow \mathbf{R}$ be a $C^{1}$ functional with compact derivative. Assume that

$$
\lim _{\|x\| \rightarrow+\infty}(\Phi(x)-\lambda \Psi(x))=+\infty
$$

for all $\lambda \in I$ and that there exists $\rho \in \mathbf{R}$ such that

$$
\sup _{\lambda \in I} \inf _{x \in X}(\Phi(x)+\lambda(\rho-\Psi(x)))<\inf _{x \in X} \sup _{\lambda \in I}(\Phi(x)+\lambda(\rho-\Psi(x))) .
$$

Then there exist a nonempty open set $\Lambda \subset I$ and a positive number $\varrho$ with the following property: for every $\lambda \in \Lambda$ and every $C^{1}$ functional $-J: X \rightarrow \mathbf{R}$ with compact derivative, there exists $\mu^{*}>0$ such that, for each $\mu \in\left[0, \mu^{*}\right]$, the equation

$$
\Phi^{\prime}(x)-\lambda \Psi^{\prime}(x)-\mu J^{\prime}(x)=0
$$

has at least three solutions in $X$ with norms less than $\varrho$. 
Proposition 2.3 (see [41]) Let $X$ be a nonempty set, and let $\Phi, \Psi$ be real functions on $X$. Assume that there are $r>0$ and $x_{0}, x_{1} \in X$ such that

$$
\Phi\left(x_{0}\right)=\Psi\left(x_{0}\right)=0, \quad \Phi\left(x_{1}\right)>r, \quad \sup _{\left.x \in \Phi^{-1}((-\infty, r)]\right)} \Psi(x)<r \frac{\Psi\left(x_{1}\right)}{\Phi\left(x_{1}\right)} .
$$

Then for each $\rho$ satisfying

$$
\sup _{\left.x \in \Phi^{-1}((-\infty, r)]\right)} \Psi(x)<\rho<r \frac{\Psi\left(x_{1}\right)}{\Phi\left(x_{1}\right)}
$$

we have

$$
\sup _{\lambda \geq 0} \inf _{x \in X}(\Phi(x)+\lambda(\rho-\Psi(x)))<\inf _{x \in X} \sup _{\lambda \geq 0}(\Phi(x)+\lambda(\rho-\Psi(x))) .
$$

Let $C_{0}^{\infty}\left([0, T], \mathbf{R}^{\mathbf{N}}\right)$ be the set of all functions $x \in C_{0}^{\infty}\left([0, T], \mathbf{R}^{\mathbf{N}}\right)$ with $x(0)=x(T)=0$ and the norm

$$
\|x\|_{\infty}=\max _{[0, T]}|x(t)| .
$$

Denote the norm of the space $L^{p}\left([0, T], \mathbf{R}^{\mathbf{N}}\right)$ for $1 \leq p<\infty$ by

$$
\|x\|_{L^{p}}=\left(\int_{0}^{T}|x(s)|^{p} d s\right)^{1 / p}
$$

The following lemma shows the boundedness of the Riemann-Liouville fractional integral operators from the space $L^{p}\left([0, T], \mathbf{R}^{\mathbf{N}}\right)$ to the space $L^{p}\left([0, T], \mathbf{R}^{\mathbf{N}}\right)$, where $1 \leq p<\infty$.

Lemma 2.4 ([20]) Let $0<\alpha \leq 1$ and $1 \leq p<\infty$. Then, for any $f \in L^{p}\left([0, T], \mathbf{R}^{\mathbf{N}}\right)$,

$$
\left\|{ }_{0} D_{\xi}^{-\alpha} f\right\|_{L^{p}([0, t])} \leq \frac{t^{\alpha}}{\Gamma(\alpha+1)}\|f\|_{L^{p}([0, t])} \quad \text { for } \xi \in[0, t], t \in[0, T]
$$

where ${ }_{0} D_{t}^{-\alpha}$ is left Riemann-Liouville fractional integral of order $\alpha$, and $\Gamma$ is the gamma function.

Definition 2.5 Let $0<\alpha \leq 1$. The fractional derivative space $E_{0}^{\alpha}$ is defined by the closure of $C_{0}^{\infty}([0, T], \mathbf{R})$ with respect to the weighted norm

$$
\|u\|_{\alpha}=\left(\int_{0}^{T} a(t)\left|{ }_{0} D_{t}^{\alpha} u(t)\right|^{2} d t+\int_{0}^{T}|u(t)|^{2} d t\right)^{1 / 2}, \quad \forall u \in E_{0}^{\alpha}
$$

Clearly, $E_{0}^{\alpha}$ is the space of functions $u \in L^{2}[0, T]$ having an $\alpha$-order fractional derivative ${ }_{0} D_{t}^{\alpha} u \in L^{2}[0, T]$ and $u(0)=u(T)=0$. From [20, Proposition 3.1] we know that, for $0<\alpha \leq$ 1 , the space $E_{0}^{\alpha}$ is a reflexive separable Banach space.

Lemma 2.6 ([31]) Let $0<\alpha \leq 1$. For any $u \in E_{0}^{\alpha}$, we have 
(i)

$$
\|u\|_{L^{2}} \leq \frac{T^{\alpha}}{\Gamma(\alpha+1) \sqrt{a_{0}}}\left(\int_{0}^{T} a(t)\left|{ }_{0} D_{t}^{\alpha} u(t)\right|^{2} d t\right)^{1 / 2}
$$

(ii) if $\alpha>\frac{1}{2}$, then

$$
\|u\|_{\infty} \leq \frac{T^{\alpha-\frac{1}{2}}}{\Gamma(\alpha) \sqrt{a_{0}(2 \alpha-1)}}\left(\int_{0}^{T} a(t)\left|{ }_{0} D_{t}^{\alpha} u(t)\right|^{2}\right)^{1 / 2} .
$$

By (2.3) we can take $E_{0}^{\alpha}$ with the norm

$$
\|u\|_{\alpha}=\left(\left.\left.\int_{0}^{T} a(t)\right|_{0} D_{t}^{\alpha} u(t)\right|^{2} d t\right)^{1 / 2}, \quad \forall u \in E_{0}^{\alpha},
$$

in the following literature.

Similarly to [20, Proposition 3.3], the space $E_{0}^{\alpha}$ (or $E_{0}^{\beta}$ ) possesses the following property:

Lemma 2.7 Assume that $\frac{1}{2}<\alpha \leq 1$ and the sequence $\left\{u_{n}\right\}$ converges weakly to $u$ in $E_{0}^{\alpha}$ : $u_{k} \rightarrow u$ in $C([0, T], \mathbf{R})$, that is, $\left\|u_{k}-u\right\|_{\infty} \rightarrow 0$ as $k \rightarrow \infty$.

We further denote by $X$ the space $E_{0}^{\alpha} \times E_{0}^{\beta}$, which is a reflexive Banach space endowed with the norm

$$
\|(u, v)\|_{X}=\|u\|_{\alpha}+\|v\|_{\beta} .
$$

Obviously, $X$ is compactly embedded in $C^{0}([0, T], \mathbf{R}) \times C^{0}([0, T], \mathbf{R})$.

Definition 2.8 By a weak solution of problem $\left(P_{\lambda, \mu}\right)$ we mean any $(u, v) \in X$ such that

$$
\begin{aligned}
\int_{0}^{T} a(t)_{0} D_{t}^{\alpha} u(t)_{0} D_{t}^{\alpha} x(t) d t+\int_{0}^{T} b(t)_{0} D_{t}^{\beta} v(t)_{0} D_{t}^{\beta} y(t) d t \\
\quad-\int_{0}^{T} h_{1}(u(t)) x(t) d t-\int_{0}^{T} h_{2}(v(t)) y(t) d t \\
=\lambda \int_{0}^{T}\left(F_{u}(t, u(t), v(t)) x(t)+F_{\nu}(t, u(t), v(t)) y(t)\right) d t \\
\quad+\mu \int_{0}^{T}\left(G_{u}(t, u(t), v(t)) x(t)+G_{v}(t, u(t), v(t)) y(t)\right) d t
\end{aligned}
$$

for every $(x, y) \in X$.

To investigate problem $\left(P_{\lambda, \mu}\right)$, we define the functionals $\Phi, \Psi, J: X \rightarrow \mathbf{R}$ by

$$
\begin{aligned}
& \Phi(u, v):=\frac{1}{2}\|u\|_{\alpha}^{2}+\frac{1}{2}\|v\|_{\beta}^{2}-H(u, v), \\
& \Psi(u, v):=\int_{0}^{T} F(t, u(t), v(t)) d t, \quad J(u, v)=\int_{0}^{T} G(t, u(t), v(t)) d t
\end{aligned}
$$


where

$$
H(u, v)=\int_{0}^{T} H_{1}(u(t)) d t+\int_{0}^{T} H_{2}(v(t)) d t, \quad H_{i}(z)=\int_{0}^{z} h_{i}(s) d s, \quad i=1,2 .
$$

Clearly, $\Psi$ and $J$ are well-defined continuously Gâteaux-differentiable functional at any $(u, v) \in X$, and their Gâteaux derivatives are

$$
\begin{aligned}
& \Psi^{\prime}(u, v)(x, y)=\int_{0}^{T}\left(F_{u}(t, u(t), v(t)) x(t)+F_{v}(t, u(t), v(t)) y(t)\right) d t, \\
& J^{\prime}(u, v)(x, y)=\int_{0}^{T}\left(G_{u}(t, u(t), v(t)) x(t)+G_{v}(t, u(t), v(t)) y(t)\right) d t,
\end{aligned}
$$

respectively, for every $(x, y) \in X$.

For convenience, put

$$
\begin{aligned}
& \rho:=\max \left\{1+\frac{L_{1} T^{2 \alpha}}{(\Gamma(\alpha+1))^{2} a_{0}}, 1+\frac{L_{2} T^{2 \beta}}{(\Gamma(\beta+1))^{2} b_{0}}\right\}, \\
& \kappa:=\min \left\{1-\frac{L_{1} T^{2 \alpha}}{(\Gamma(\alpha+1))^{2} a_{0}}, 1-\frac{L_{2} T^{2 \beta}}{(\Gamma(\beta+1))^{2} b_{0}}\right\} .
\end{aligned}
$$

We introduce the following hypothesis:

(H1) $\frac{1}{2}<\alpha, \beta \leq 1$ and $\kappa>0$.

Lemma 2.9 The functional $\Phi$ is sequentially weakly lower semicontinuous and bounded on $X$, and $\Phi^{\prime}$ admits a continuous inverse on $X^{*}$.

Proof Let $\left\{\left(u_{n}, v_{n}\right)\right\} \subset X,\left(u_{n}, v_{n}\right) \rightarrow(u, v)$ in $X$. From Lemma 2.7, $\left(u_{n}, v_{n}\right)$ converges uniformly to $(u, v)$ on $[0, T]$, and $\lim _{n \rightarrow \infty} \inf \left\|\left(u_{n}, v_{n}\right)\right\|_{X} \geq\|(u, v)\|_{X}$. Thus

$$
\begin{aligned}
\liminf _{n \rightarrow \infty} \Phi\left(u_{n}, v_{n}\right) & =\liminf _{n \rightarrow \infty}\left(\frac{1}{2}\left\|u_{n}\right\|_{\alpha}^{2}+\frac{1}{2}\left\|v_{n}\right\|_{\beta}^{2}\right)-\lim _{n \rightarrow \infty} H\left(u_{n}, v_{n}\right) \\
& \geq \frac{1}{2}\|u\|_{\alpha}^{2}+\frac{1}{2}\|v\|_{\beta}^{2}-H(u, v)=\Phi(u, v) .
\end{aligned}
$$

So $\Phi$ is a sequentially weakly lower semicontinuous functional.

Since $h_{1}, h_{2}$ are Lipschitz continuous and satisfy $h_{1}(0)=h_{2}(0)$, we have $\left|h_{i}(s)\right| \leq L_{i}|s|$, $i=1,2$, for all $s \in \mathbf{R}$. Moreover, let $\Omega$ be a bounded subset of $X$, that is, there is a constant $c>0$ such that $\|(u, v)\|_{X} \leq c$ for any $(u, v) \in \Omega$. By (2.3) and Lemma 2.7 we have

$$
\begin{aligned}
\Phi(u, v) & \leq \frac{1}{2}\|u\|_{\alpha}^{2}+\frac{1}{2}\|v\|_{\beta}^{2}+\left|\int_{0}^{T} H_{1}(u(t)) d t\right|+\left|\int_{0}^{T} H_{2}(v(t)) d t\right| \\
& \leq \frac{1}{2}\|u\|_{\alpha}^{2}+\frac{1}{2}\|v\|_{\beta}^{2}+\frac{L_{1}}{2} \int_{0}^{T}|u(t)|^{2} d t+\frac{L_{2}}{2} \int_{0}^{T}|v(t)|^{2} d t \\
& \leq\left(\frac{1}{2}+\frac{L_{1} T^{2 \alpha}}{2(\Gamma(\alpha+1))^{2} a_{0}}\right)\|u\|_{\alpha}^{2}+\left(\frac{1}{2}+\frac{L_{2} T^{2 \beta}}{2(\Gamma(\beta+1))^{2} b_{0}}\right)\|v\|_{\beta}^{2}
\end{aligned}
$$




$$
\begin{aligned}
& \leq \frac{\rho}{2}\left(\|u\|_{\alpha}^{2}+\|v\|_{\beta}^{2}\right) \\
& \leq \frac{\rho c^{2}}{2} .
\end{aligned}
$$

Hence $\Phi$ is bounded on each bounded subset of $X$.

Next, we will show that $\Phi^{\prime}: X \rightarrow X^{*}$ admits a Lipschitz continuous inverse. Obviously, $\Phi \in C^{1}(X, \mathbf{R})$ and

$$
\begin{aligned}
\left\langle\Phi^{\prime}(u, v),(x, y)\right\rangle= & \int_{0}^{T} a(t)_{0} D_{t}^{\alpha} u(t)_{0} D_{t}^{\alpha} x(t) d t+\int_{0}^{T} b(t)_{0} D_{t}^{\beta} v(t)_{0} D_{t}^{\beta} y(t) d t \\
& -\int_{0}^{T} h_{1}(u(t)) x(t) d t-\int_{0}^{T} h_{2}(v(t)) y(t) d t \\
= & \left\langle\Phi_{1}(u), x\right\rangle+\left\langle\Phi_{2}(v), y\right\rangle,
\end{aligned}
$$

where

$$
\begin{aligned}
& \left\langle\Phi_{1}(u), x\right\rangle=\int_{0}^{T} a(t)_{0} D_{t}^{\alpha} u(t)_{0} D_{t}^{\alpha} x(t) d t-\int_{0}^{T} h_{1}(u(t)) x(t) d t, \quad \forall x \in E_{0}^{\alpha}, \\
& \left\langle\Phi_{2}(v), y\right\rangle=\int_{0}^{T} b(t)_{0} D_{t}^{\beta} v(t)_{0} D_{t}^{\beta} y(t) d t-\int_{0}^{T} h_{2}(v(t)) y(t) d t, \quad \forall y \in E_{0}^{\beta} .
\end{aligned}
$$

For any $u, x \in E_{0}^{\alpha}$, it follows from (1.2), (2.3), and (2.11) that

$$
\begin{aligned}
\left\langle\Phi_{1}(u)-\Phi_{1}(x), u-x\right\rangle= & \int_{0}^{T} a(t)\left({ }_{0} D_{t}^{\alpha}(u(t)-x(t))\right)^{2} d t \\
& -\int_{0}^{T}\left(h_{1}(u(t))-h_{1}(x(t))\right)(u(t)-x(t)) d t \\
\geq & \int_{0}^{T} a(t)\left({ }_{0} D_{t}^{\alpha}(u(t)-x(t))\right)^{2} d t-L_{1} \int_{0}^{T}(u(t)-x(t))^{2} d t \\
\geq & \left(1-\frac{L_{1} T^{2 \alpha}}{(\Gamma(\alpha+1))^{2} a_{0}}\right)\|u-x\|_{\alpha}^{2} \geq \kappa\|u-x\|_{\alpha}^{2} .
\end{aligned}
$$

Thus we deduce from the assumption $\kappa>0$ that $\Phi_{1}$ is a uniformly monotone operator. Similarly, it is easy to show that $\Phi_{2}$ is also a uniformly monotone operator. So $\Phi^{\prime}$ is uniformly monotone.

For any $(u, v) \in X \backslash\{(0,0)\}$, we have

$$
\begin{aligned}
& \frac{\left\langle\Phi^{\prime}(u, v),(u, v)\right\rangle}{\|(u, v)\|_{X}} \\
& \quad=\frac{\int_{0}^{T}\left(\left.\left.a(t)\right|_{0} D_{t}^{\alpha} u(t)\right|^{2} d t+\left.\left.b(t)\right|_{0} D_{t}^{\beta} u(t)\right|^{2}\right) d t-\int_{0}^{T}\left(h_{1}(u(t)) u(t)+h_{2}(v(t)) v(t)\right) d t}{\|u\|_{\alpha}+\|v\|_{\beta}} \\
& \quad \geq \frac{\kappa\left(\|u\|_{\alpha}^{2}+\|v\|_{\beta}^{2}\right)}{\|u\|_{\alpha}+\|v\|_{\beta}},
\end{aligned}
$$

which implies that

$$
\lim _{\|(u, v)\|_{X} \rightarrow \infty} \frac{\left\langle\Phi^{\prime}(u, v),(u, v)\right\rangle}{\|(u, v)\|_{X}}=+\infty
$$


since $\lim _{x+y \rightarrow+\infty} \frac{x^{2}+y^{2}}{x+y}=+\infty, x, y \geq 0$. It follows from the Minty-Browder theorem [42, Theorem 26.A] that $\Phi^{\prime}$ is a Lipschitz continuous inverse on $X^{*}$.

Lemma 2.10 The functionals $\Psi$ and J are continuously Gâteaux differentiable in $X$, and their derivatives $\Psi^{\prime}$, $J^{\prime}$ are compact.

Proof We claim that the functional $\Psi$ is sequentially weakly upper semicontinuous and $\Psi^{\prime}: X \rightarrow X^{*}$ is a compact operator. Indeed, for fixed $(u, v) \in X$, suppose that $\left\{\left(u_{n}, v_{n}\right)\right\} \subset X$, $\left(u_{n}, v_{n}\right) \rightarrow(u, v)$ in $X$ as $n \rightarrow+\infty$. Then $\left(u_{n}, v_{n}\right)$ converges uniformly to $(u, v)$ on $[0, T]$. Hence

$$
\lim _{n \rightarrow+\infty} \sup \Psi\left(u_{n}, v_{n}\right) \leq \int_{0}^{T} \lim \sup _{n \rightarrow+\infty} F\left(t, u_{n}, v_{n}\right) d t=\int_{0}^{T} F(t, u, v) d t=\Psi(u, v)
$$

which yields that $\Psi$ is sequentially weakly upper semicontinuous. On the other hand, taking into account that $F(t, \cdot, \cdot)$ is a $C^{1}$ function in $\mathbf{R}^{2}$ for all $t \in[0, T]$, so it is continuous in $\mathbf{R}^{2}$ for all $t \in[0, T]$, and we have $F\left(t, u_{n}, v_{n}\right) \rightarrow F(t, u, v)$ as $n \rightarrow+\infty$. By the Lebesgue convergence theorem, $\Psi^{\prime}\left(u_{n}, v_{n}\right) \rightarrow \Psi^{\prime}(u, v)$ strongly, which implies that $\Psi^{\prime}$ is strongly continuous on $X$. Therefore $\Psi^{\prime}$ is a compact operator.

Analogously, we can deduce that $J^{\prime}(u, v)$ is a compact operator for any $(u, v) \in X$.

Similarly to the proof of [21, Theorem 5.1$]$, we have the following:

Lemma 2.11 Let $\frac{1}{2}<\alpha, \beta \leq 1$ and $(u, v) \in X$. If $(u, v)$ is a nontrivial weak solution of problem $\left(P_{\lambda, \mu}\right)$, then $(u, v)$ is also a nontrivial solution of problem $\left(P_{\lambda, \mu}\right)$.

\section{Main results and proof}

In this section, we investigate the existence of at least three weak solutions for problem $\left(P_{\lambda, \mu}\right)$. For the convenience of the reader, put

$$
\begin{aligned}
M= & \max \left\{\frac{T^{2 \alpha-1}}{(\Gamma(\alpha))^{2} a_{0}(2 \alpha-1)}, \frac{T^{2 \beta-1}}{(\Gamma(\beta))^{2} b_{0}(2 \beta-1)}\right\}, \\
\lambda_{1}= & \inf \left\{\frac{\|u\|_{\alpha}^{2}+\|v\|_{\beta}^{2}+2 H(u, v)}{2 \int_{0}^{T} F(t, u, v) d t},(u, v) \in X, \int_{0}^{T} F(t, u, v) d t>0\right\}, \\
\lambda_{2}= & \left(\operatorname { m a x } \left\{0, \limsup _{\|(u, v)\|_{X} \rightarrow+\infty} \frac{2 \int_{0}^{T} F(t, u, v) d t}{\|u\|_{\alpha}^{2}+\|v\|_{\beta}^{2}-2 H(u, v)},\right.\right. \\
& \left.\left.\limsup _{(u, v) \rightarrow 0} \frac{2 \int_{0}^{T} F(t, u, v) d t}{\|u\|_{\alpha}^{2}+\|v\|_{\beta}^{2}-2 H(u, v)}\right\}\right)^{-1} .
\end{aligned}
$$

For a given constant $\epsilon \in\left(0, \frac{1}{2}\right)$, set

$$
\begin{aligned}
A(\alpha, \epsilon)= & \frac{1}{2 \epsilon^{2} T^{2}}\left\{\int_{0}^{T} a(t) t^{2(1-\alpha)} d t+\int_{\epsilon T}^{T} a(t)(t-\epsilon T)^{2(1-\alpha)} d t\right. \\
& +\int_{(1-\epsilon) T}^{T} a(t)(t-(1-\epsilon) T)^{2(1-\alpha)} d t-2 \int_{(1-\epsilon) T}^{T} a(t)\left(t^{2}-(1-\epsilon) T t\right)^{1-\alpha} d t \\
& \left.-2 \int_{\epsilon T}^{T} a(t)\left(t^{2}-\epsilon T t\right)^{1-\alpha} d t+2 \int_{(1-\epsilon) T}^{T} a(t)\left(t^{2}-\epsilon T t+\theta(1-\epsilon) T^{2}\right)^{1-\alpha} d t\right\}
\end{aligned}
$$


and

$$
\begin{aligned}
& B(\beta, \epsilon)= \frac{1}{2 \epsilon^{2} T^{2}}\left\{\int_{0}^{T} b(t) t^{2(1-\beta)} d t+\int_{\epsilon T}^{T} b(t)(t-\epsilon T)^{2(1-\beta)} d t\right. \\
&+\int_{(1-\epsilon) T}^{T} b(t)(t-(1-\epsilon) T)^{2(1-\beta)} d t-2 \int_{(1-\epsilon) T}^{T} b(t)\left(t^{2}-(1-\epsilon) T t\right)^{1-\beta} d t \\
&\left.-2 \int_{\epsilon T}^{T} b(t)\left(t^{2}-\epsilon T t\right)^{1-\beta} d t+2 \int_{(1-\epsilon) T}^{T} b(t)\left(t^{2}-T t+\epsilon(1-\epsilon) T^{2}\right)^{1-\beta} d t\right\}, \\
& \triangle_{1}:=\min \{A(\alpha, \epsilon), B(\beta, \epsilon)\}, \quad \triangle_{2}:=\max \{A(\alpha, \epsilon), B(\beta, \epsilon)\} .
\end{aligned}
$$

For any $d>0$, we denote by $\Omega(d)$ the set

$$
\left\{(x, y) \in \mathbf{R}^{2}:|x|^{2}+|y|^{2} \leq d\right\} .
$$

Theorem 3.1 Assume that (F0), (H0), and (H1) hold. Moreover, assume that there exist a constant $\eta \geq 0$ and a function $\bar{\omega}=\left(\omega_{1}, \omega_{2}\right) \in X$ such that

(B1)

$$
\max \left\{\limsup _{(u, v) \rightarrow(0,0)} \frac{\max _{t \in[0, T]} F(t, u, v)}{|u|^{2}+|v|^{2}}, \limsup _{|(u, v)| \rightarrow+\infty} \frac{\max _{t \in[0, T]} F(t, u, v)}{|u|^{2}+|v|^{2}}\right\} \leq \eta
$$

$$
\frac{2 T M \eta}{\kappa}<\frac{\int_{0}^{T} F\left(t, \omega_{1}(t), \omega_{2}(t)\right) d t}{\rho\left(\left\|\omega_{1}\right\|_{\alpha}^{2}+\left\|\omega_{2}\right\|_{\beta}^{2}\right)} .
$$

Then, for any compact interval $\left[a_{1}, a_{2}\right] \subset\left(\lambda_{1}, \lambda_{2}\right)$, there exists a positive constant $\varrho$ with the following property: for every $\lambda \in\left[a_{1}, a_{2}\right]$ and for two Carathéodory functions $G_{u}, G_{v}$ satisfying $(G 0)$, there is $\delta>0$ such that, for each $\mu \in[0, \delta)$, problem $\left(P_{\lambda, \mu}\right)$ has at least three weak solutions with norms less than $\varrho$.

Proof Our aim is to apply Theorem 2.1 to our problem $\left(P_{\lambda, \mu}\right)$ by taking $X=E_{0}^{\alpha} \times E_{0}^{\beta}$ endowed with the norm $\|(u, v)\|_{X}$ defined before. Obviously, $X$ is a separable reflexive Banach space. It follows from Lemmas 2.9 and 2.10 that the functional $\Phi$ is sequentially weakly lower semicontinuous, with continuous Gâteaux derivative, and bounded on each bounded subset of $X . \Phi^{\prime}$ admits a continuous inversem and $\Psi$ and $J$ are continuously Gâteaux-differentiable functionals in $X$ with compact derivatives.

It is easy to see that $\frac{1}{2}\|u\|_{\alpha}^{2}+\frac{1}{2}\|v\|_{\beta}^{2}$ belongs to $\Upsilon_{X}$ (see the beginning of Sect. 2). Now we prove that $\Phi(u, v) \in \Upsilon_{X}$. Let $\left\{\left(u_{n}, v_{n}\right)\right\} \subset X,\left(u_{n}, v_{n}\right) \rightarrow(u, v)$ in $X$, and $\lim _{n \rightarrow \infty} \inf \Phi\left(u_{n}\right.$, $\left.v_{n}\right) \leq \Phi(u, v)$. By Lemma 2.7, $\left(u_{n}, v_{n}\right)$ converges uniformly to $(u, v)$ on $[0, T]$. Thus there exist constants $c_{1}, c_{2}>0$ such that $\left\|u_{n}\right\|_{\infty} \leq c_{1}$ and $\left\|v_{n}\right\|_{\infty} \leq c_{2}$ for any $n \in \mathbf{N}$. Then

$$
\left|H_{1}\left(u_{n}(t)\right)-H_{1}(u(t))\right| \leq L_{1}\left|\int_{u(t)}^{u_{n}(t)}\right| s|d s| \leq \frac{L_{1}}{2}\left(\left|u_{n}(t)\right|^{2}+|u(t)|^{2}\right) \leq \frac{L_{1}}{2}\left(c_{1}^{2}+\|u\|_{\infty}^{2}\right)
$$

and

$$
\left|H_{2}\left(v_{n}(t)\right)-H_{2}(v(t))\right| \leq L_{2}\left|\int_{v(t)}^{v_{n}(t)}\right| s|d s| \leq \frac{L_{2}}{2}\left(\left|v_{n}(t)\right|^{2}+|v(t)|^{2}\right) \leq \frac{L_{2}}{2}\left(c_{2}^{2}+\|v\|_{\infty}^{2}\right)
$$


for any $n \in \mathbf{N}$ and $t \in[0, T]$. Furthermore, $H_{1}\left(u_{n}(t)\right) \rightarrow H_{1}(u(t))$ and $H_{2}\left(v_{n}(t)\right) \rightarrow H_{2}(v(t))$ at every $t \in[0, T]$, and by the Lebesgue convergence theorem

$$
\begin{aligned}
H\left(u_{n}, v_{n}\right) & =\int_{0}^{T} H_{1}\left(u_{n}(t)\right) d t+\int_{0}^{T} H_{2}\left(v_{n}(t)\right) d t \\
& \rightarrow \int_{0}^{T} H_{1}(u(t)) d t+\int_{0}^{T} H_{2}(v(t)) d t \\
& =H(u, v),
\end{aligned}
$$

which yields that $\liminf _{n \rightarrow \infty} H\left(u_{n}, v_{n}\right) \leq H(u, v)$. Thus, $\left\{\left(u_{n}, v_{n}\right)\right\}$ has a subsequence converging strongly to $(u, v)$. Therefore $\Phi \in \Upsilon_{X}$.

Due to assumption (1.2), we infer that

$$
\begin{aligned}
\Phi(u, v) & \geq \frac{1}{2}\|u\|_{\alpha}^{2}+\frac{1}{2}\|v\|_{\beta}^{2}-\left|\int_{0}^{T} H_{1}(u(t)) d t\right|-\left|\int_{0}^{T} H_{2}(v(t)) d t\right| \\
& \geq\left(\frac{1}{2}-\frac{L_{1} T^{2 \alpha}}{2(\Gamma(\alpha+1))^{2} a_{0}}\right)\|u\|_{\alpha}^{2}+\left(\frac{1}{2}-\frac{L_{2} T^{2 \beta}}{2(\Gamma(\beta+1))^{2} b_{0}}\right)\|v\|_{\beta}^{2} \\
& \geq \frac{\kappa}{2}\left(\|u\|_{\alpha}^{2}+\|v\|_{\beta}^{2}\right)
\end{aligned}
$$

for all $(u, v) \in X$. So $\Phi$ is coercive and has a strict local minimum $\left(u_{0}, v_{0}\right)=(0,0)$ with $\Phi\left(u_{0}, v_{0}\right)=\Psi\left(u_{0}, v_{0}\right)=0$.

Fix $\varepsilon>0$. According to (B1), there exist $\sigma_{1}, \sigma_{2}$ with $0<\sigma_{1}<\sigma_{2}$ such that

$$
F(t, u, v) \leq(\eta+\varepsilon)\left(|u|^{2}+|v|^{2}\right)
$$

for all $t \in[0, T]$ and $|(u, v)| \in\left(\left[0, \sigma_{1}\right) \cup\left(\sigma_{2},+\infty\right)\right)$. In view of (F0), $F(t, u, v)$ is bounded on $t \in[0, T]$ and $|(u, v)| \in\left[\sigma_{1}, \sigma_{2}\right]$, so we can choose $p_{1}, p_{2}>0$ and $\tau_{1}, \tau_{2}>2$ such that

$$
F(t, u, v) \leq(\eta+\varepsilon)\left(|u|^{2}+|v|^{2}\right)+p_{1}|u|^{\tau_{1}}+p_{2}|v|^{\tau_{2}}
$$

for all $t \in[0, T]$ and $|(u, v)| \in\left[\sigma_{1}, \sigma_{2}\right]$. So, from (2.9) we have

$$
\begin{aligned}
\Psi(u, v) & \leq(\eta+\varepsilon) \int_{0}^{T}\left(|u|^{2}+|v|^{2}\right) d t+\int_{0}^{T}\left(p_{1}|u|^{\tau_{1}}+p_{2}|v|^{\tau_{2}}\right) d t \\
& \leq(\eta+\varepsilon) T M\left(\|u\|_{\alpha}^{2}+\|v\|_{\beta}^{2}\right)+T \zeta\left(\|u\|_{\alpha}^{\tau_{1}}+\|v\|_{\beta}^{\tau_{2}}\right)
\end{aligned}
$$

for all $(u, v) \in X$, where

$$
\zeta=\max \left\{p_{1}\left(\frac{T^{\alpha-\frac{1}{2}}}{\Gamma(\alpha) \sqrt{a_{0}(2 \alpha-1)}}\right)^{\tau_{1}}, p_{2}\left(\frac{T^{\beta-\frac{1}{2}}}{\Gamma(\beta) \sqrt{b_{0}(2 \beta-1)}}\right)^{\tau_{2}}\right\} .
$$

Hence

$$
\limsup _{(u, v) \rightarrow 0} \frac{\Psi(u, v)}{\Phi(u, v)} \leq \frac{2 T M(\eta+\varepsilon)}{\kappa} .
$$


Furthermore, by (3.4) again, for any $(u, v) \in X \backslash\{(0,0)\}$, we have

$$
\begin{aligned}
\frac{\Psi(u, v)}{\Phi(u, v)} & =\frac{\int_{|(u, v)| \leq \sigma_{2}} F(t, u, v) d t}{\frac{1}{2}\|u\|_{\alpha}^{2}+\frac{1}{2}\|v\|_{\beta}^{2}-H(u, v)}+\frac{\int_{|(u, v)|>\sigma_{2}} F(t, u, v) d t}{\frac{1}{2}\|u\|_{\alpha}^{2}+\frac{1}{2}\|v\|_{\beta}^{2}-H(u, v)} \\
& \leq \frac{2 T \sup _{t \in[0, T],|(u, v)| \in\left[0, \sigma_{2}\right]} F(t, u, v)}{\kappa\left(\|u\|_{\alpha}^{2}+\|v\|_{\beta}^{2}\right)}+\frac{2 T M(\eta+\varepsilon)\left(\|u\|_{\alpha}^{2}+\|v\|_{\beta}^{2}\right)}{\kappa\left(\|u\|_{\alpha}^{2}+\|v\|_{\beta}^{2}\right)} \\
& =\frac{2 T \sup _{t \in[0, T],|(u, v)| \in\left[0, \sigma_{2}\right]} F(t, u, v)}{\kappa\left(\|u\|_{\alpha}^{2}+\|v\|_{\beta}^{2}\right)}+\frac{2 T M(\eta+\varepsilon)}{\kappa},
\end{aligned}
$$

which implies that

$$
\lim _{\|(u, v)\|_{X} \rightarrow+\infty} \frac{\Psi(u, v)}{\Phi(u, v)} \leq \frac{2 T M(\eta+\varepsilon)}{\kappa} .
$$

Since $\varepsilon$ is arbitrary, combining with (3.5) and (3.6), we have

$$
\delta_{1}=\max \left\{0, \lim _{(u, v) \rightarrow 0} \frac{\Psi(u, v)}{\Phi(u, v)}, \lim _{\|(u, v)\|_{X} \rightarrow+\infty} \frac{\Psi(u, v)}{\Phi(u, v)}\right\} \leq \frac{2 T M \eta}{\kappa}
$$

and

$$
\begin{aligned}
\delta_{2} & =\sup _{(u, v) \in \Phi^{-1}((0,+\infty))} \frac{\Psi(u, v)}{\Phi(u, v)}=\sup _{(u, v) \in X \backslash\{(0,0)\}} \frac{\Psi(u, v)}{\Phi(u, v)} \\
& \geq \frac{\int_{0}^{T} F\left(t, \omega, \omega_{2}\right) d t}{\frac{1}{2}\left\|\omega_{1}\right\|_{\alpha}^{2}+\frac{1}{2}\left\|\omega_{2}\right\|_{\beta}^{2}+H\left(\omega_{1}, \omega_{2}\right)} \\
& \geq \frac{2 \int_{0}^{T} F\left(t, \omega, \omega_{2}\right) d t}{\rho\left(\left\|\omega_{1}\right\|_{\alpha}^{2}+\left\|\omega_{2}\right\|_{\beta}^{2}\right)} \\
& >\frac{2 T M \eta}{\kappa} \geq \delta_{1} .
\end{aligned}
$$

Then, for each compact interval $\left[a_{1}, a_{2}\right] \subset\left(\lambda_{1}, \lambda_{2}\right)$, there is $\varrho>0$ with the following property: for all $\lambda \in\left[a_{1}, a_{2}\right]$ and $G \in(G 0)$, there exists $\delta>0$ such that for $\mu \in[0, \delta]$, problem $\left(P_{\lambda, \mu}\right)$ has at least three weak solutions with norms less than $\varrho$.

Theorem 3.2 Assume that (F0), (H0), and (H1) hold and there exist $l, q \in L^{1}\left([0, T], \mathbf{R}^{+}\right)$, three positive constants $d, \theta_{1}, \theta_{2}$, and constant vector $\bar{c}=\left(c_{1}, c_{2}\right) \in \mathbf{R}^{2}, c_{1}, c_{2}>0$, with $d<$ $2 M \triangle_{1}\left(c_{1}^{2}+c_{2}^{2}\right)$ and $\theta_{1}, \theta_{2} \in[0,2)$, such that

(B3) $F(t, u, v) \geq 0$ for all $t \in[0, \epsilon T] \cup[(1-\epsilon) T, T],|u| \leq \Gamma(2-\alpha) c_{1}$, and $|v| \leq \Gamma(2-\beta) c_{2}$

(B4) $|F(t, u, v)| \leq l(t)\left(|u|^{\theta_{1}}+|v|^{\theta_{2}}\right)+q(t)$ for every $(u, v) \in X$ and a.e. $t \in[0, T]$;

(B5)

$$
\max _{t \in[0, T],(u, v) \in \Omega(d)} F(t, u, v)<\frac{d \kappa}{2 M T} \cdot \frac{\int_{\epsilon T}^{(1-\epsilon) T} F\left(t, \Gamma(2-\alpha) c_{1}, \Gamma(2-\beta) c_{2}\right) d t}{\rho \triangle_{2}\left(c_{1}^{2}+c_{2}^{2}\right)}
$$

where $\Omega(d)=\left\{(\xi, \eta) \in \mathbf{R}^{2}:|u|^{2}+|v|^{2} \leq d\right\}$. 
Then there exist an open interval $\Lambda \subset[0,+\infty)$ and a positive constant $\varrho$ with the following property: for every $\lambda \in \Lambda$ and for two Carathéodory functions $G_{u}, G_{v}$ satisfying (G0), there is $\delta>0$ such that, for each $\mu \in[0, \delta)$, problem $\left(P_{\lambda, \mu}\right)$ has at least three weak solutions with norms less than $\varrho$.

Proof For any $\lambda \geq 0$ and $(u, v) \in X$, according to (3.3) and (B4), we have

$$
\begin{aligned}
\Phi(u, v)-\lambda \Psi(u, v)= & \frac{1}{2}\|u\|_{\alpha}^{2}+\frac{1}{2}\|v\|_{\beta}^{2}-H(u, v)-\lambda \int_{0}^{T} F(t, u, v) d t \\
\geq & \frac{1}{2}\|u\|_{\alpha}^{2}+\frac{1}{2}\|v\|_{\beta}^{2}-H(u, v)-\lambda \int_{0}^{T} l(t)\left(|u|^{\theta_{1}}+|v|^{\theta_{2}}\right) d t \\
& -\lambda \int_{0}^{T} q(t) d t \\
\geq & \frac{\kappa}{2}\left(\|u\|_{\alpha}^{2}+\|v\|_{\beta}^{2}\right)-\lambda \theta \int_{0}^{T} l(t) d t\left(\|u\|_{\alpha}^{\theta_{1}}+\|v\|_{\beta}^{\theta_{2}}\right) \\
& -\lambda \int_{0}^{T} q(t) d t,
\end{aligned}
$$

where

$$
\theta=\max \left\{\left(\frac{T^{\alpha-\frac{1}{2}}}{\Gamma(\alpha) \sqrt{a_{0}(2 \alpha-1)}}\right)^{\theta_{1}},\left(\frac{T^{\beta-\frac{1}{2}}}{\Gamma(\beta) \sqrt{b_{0}(2 \beta-1)}}\right)^{\theta_{2}}\right\} .
$$

Since $\theta_{1}, \theta_{2} \in[0,2)$, we have

$$
\lim _{\|(u, v)\|_{X} \rightarrow+\infty}(\Phi(u, v)-\lambda \Psi(u, v))=+\infty \quad \text { for all } \lambda \geq 0 .
$$

For every $r>0$, by the definition of $\Phi$ and (3.3) we have

$$
\begin{aligned}
\Phi^{-1} & (]-\infty, r]) \\
& :=\{(u, v) \in X: \Phi(u, v) \leq r\} \\
& \subseteq\left\{(u, v) \in X:\|u\|_{\alpha}^{2}+\|v\|_{\beta}^{2} \leq \frac{2 r}{\kappa}\right\} \\
& \subseteq\left\{(u, v) \in X: \frac{(\Gamma(\alpha))^{2} a_{0}(2 \alpha-1)}{T^{2 \alpha-1}}\|u\|_{\infty}^{2}+\frac{(\Gamma(\beta))^{2} b_{0}(2 \beta-1)}{T^{2 \beta-1}}\|v\|_{\infty}^{2} \leq \frac{2 r}{\kappa}\right\} \\
& \subseteq\left\{(u, v) \in X:|u(t)|^{2}+|v(t)|^{2} \leq \frac{2 M r}{\kappa}, \text { for all } t \in[0, T]\right\} .
\end{aligned}
$$

Therefore

$$
\begin{aligned}
\sup _{\left.\left.(u, v) \in \Phi^{-1}(]-\infty, r\right]\right)} \Psi(u, v) & \leq \max _{(u, v) \in \Omega\left(\frac{2 M r}{\kappa}\right)} \Psi(u, v) \\
& =\max _{(u, v) \in \Omega\left(\frac{2 M r}{\kappa}\right)} \int_{0}^{T} F(t, u, v) d t \\
& \leq T \max _{t \in[0, T],(u, v) \in \Omega\left(\frac{2 M r}{\kappa}\right)} F(t, u, v) .
\end{aligned}
$$


Choose $\omega=\left(\omega_{1}(t), \omega_{2}(t)\right)$ with

$$
\omega_{1}(t)= \begin{cases}\frac{\Gamma(2-\alpha) c_{1}}{\epsilon T} t, & t \in[0, \epsilon T[ \\ \Gamma(2-\alpha) c_{1}, & t \in[\epsilon T,(1-\epsilon) T] \\ \frac{\Gamma(2-\alpha) c_{1}}{\epsilon T}(T-t), & t \in](1-\epsilon) T, T]\end{cases}
$$

and

$$
\omega_{2}(t)= \begin{cases}\frac{\Gamma(2-\beta) c_{2}}{\epsilon T} t, & t \in[0, \epsilon T[ \\ \Gamma(2-\beta) c_{2}, & t \in[\epsilon T,(1-\epsilon) T] \\ \frac{\Gamma(2-\beta) c_{2}}{\epsilon T}(T-t), & t \in](1-\epsilon) T, T]\end{cases}
$$

Clearly, $\omega_{i}(0)=\omega_{i}(T)=0$ and $\omega_{i} \in L^{2}[0, T]$ for $i=1$, 2. A direct calculation shows that

$$
{ }_{0} D_{t}^{\alpha} \omega_{1}(t)= \begin{cases}\frac{c_{1}}{\epsilon T} t^{1-\alpha}, & t \in[0, \epsilon T[ \\ \frac{c_{1}}{\epsilon T}\left(t^{1-\alpha}-(t-\epsilon T)^{1-\alpha}\right), & t \in[\epsilon T,(1-\epsilon) T], \\ \frac{c_{1}}{\epsilon T}\left(t^{1-\alpha}-(t-\epsilon T)^{1-\alpha}-(t-(1-\epsilon) T)^{1-\alpha}\right), & t \in](1-\epsilon) T, T],\end{cases}
$$

and

$$
{ }_{0} D_{t}^{\beta} \omega_{2}(t)= \begin{cases}\frac{c_{2}}{\epsilon T} t^{1-\beta}, & t \in[0, \epsilon T[, \\ \frac{c_{2}}{\epsilon T}\left(t^{1-\beta}-(t-\epsilon T)^{1-\beta}\right), & t \in[\epsilon T,(1-\epsilon) T], \\ \frac{c_{2}}{\epsilon T}\left(t^{1-\beta}-(t-\epsilon T)^{1-\beta}-(t-(1-\epsilon) T)^{1-\beta}\right), & t \in](1-\epsilon) T, T] .\end{cases}
$$

Furthermore,

$$
\begin{aligned}
& \left.\left.\int_{0}^{T} a(t)\right|_{0} D_{t}^{\alpha} \omega_{1}(t)\right|^{2} d t \\
& =\int_{0}^{\epsilon T}+\int_{\epsilon T}^{(1-\epsilon) T}+\int_{(1-\epsilon) T}^{T}\left(\left.\left.a(t)\right|_{0} D_{t}^{\alpha} \omega_{1}(t)\right|^{2}\right) d t \\
& =\frac{c_{1}^{2}}{\epsilon^{2} T^{2}}\left\{\int_{0}^{T} a(t) t^{2(1-\alpha)} d t+\int_{\epsilon T}^{T} a(t)(t-(1-\epsilon) T)^{2(1-\alpha)} d t\right. \\
& \quad+\int_{(1-\epsilon) T}^{T} a(t)(t-(1-\epsilon) T)^{2(1-\alpha)} d t \\
& \quad-2 \int_{\epsilon T}^{T} a(t)\left(t^{2}-(1-\epsilon) T t\right)^{1-\alpha} d t-2 \int_{(1-\epsilon) T}^{T} a(t)\left(t^{2}-(1-\epsilon) T t\right)^{1-\alpha} d t \\
& \left.\quad+2 \int_{(1-\epsilon) T}^{T} a(t)\left(t^{2}-\epsilon T t+\epsilon(1-\epsilon) T^{2} t\right)^{1-\alpha} d t\right\} \\
& =2 c_{1}^{2} A(\alpha, \epsilon)
\end{aligned}
$$


and

$$
\begin{aligned}
& \int_{0}^{T} b(t)\left|{ }_{0} D_{t}^{\beta} \omega_{2}(t)\right|^{2} d t \\
& =\int_{0}^{\epsilon T}+\int_{\epsilon T}^{(1-\epsilon) T}+\int_{(1-\epsilon) T}^{T}\left(\left.\left.b(t)\right|_{0} D_{t}^{\beta} \omega_{2}(t)\right|^{2}\right) d t \\
& =\frac{c_{2}^{2}}{\epsilon^{2} T^{2}}\left\{\int_{0}^{T} b(t) t^{2(1-\beta)} d t+\int_{\epsilon T}^{T} b(t)(t-\epsilon T)^{2(1-\beta)} d t\right. \\
& \quad+\int_{(1-\epsilon) T}^{T} b(t)(t-(1-\epsilon) T)^{2(1-\beta)} d t \\
& \quad-2 \int_{\epsilon T}^{T} b(t)\left(t^{2}-\epsilon T t\right)^{1-\beta} d t-2 \int_{(1-\epsilon) T}^{T} b(t)\left(t^{2}-(1-\epsilon) T t\right)^{1-\beta} d t \\
& \left.\quad+2 \int_{(1-\epsilon) T}^{T} a(t)\left(t^{2}-\epsilon T t+\epsilon(1-\epsilon) T^{2} t\right)^{1-\beta} d t\right\} \\
& =2 c_{2}^{2} B(\beta, \epsilon) .
\end{aligned}
$$

Thus, $\omega=\left(\omega_{1}(t), \omega_{2}(t)\right) \in X$, and

$$
2 \Delta_{1}\left(c_{1}^{2}+c_{2}^{2}\right) \leq\left\|\omega_{1}\right\|_{\alpha}^{2}+\left\|\omega_{2}\right\|_{\beta}^{2} \leq 2 \Delta_{2}\left(c_{1}^{2}+c_{2}^{2}\right) .
$$

Obviously, $\Phi(0,0)=\Psi(0,0)=0$. Choose $r=\frac{\kappa d}{2 M}$, where $M$ is given in (3.1). From $d<$ $2 M \triangle_{1}\left(c_{1}^{2}+c_{2}^{2}\right)$ and (3.7) we have

$$
2 M r=\kappa d<2 \kappa M \Delta_{1}\left(c_{1}^{2}+c_{2}^{2}\right) \leq 2 M \Phi\left(\omega_{1}, \omega_{2}\right)
$$

which means that $\Phi\left(\omega_{1}, \omega_{2}\right)>r$. According to (B3) and $F(t, 0,0)=0$, we have

$$
\int_{0}^{T} F\left(t, \omega_{1}, \omega_{2}\right) d t=\int_{0}^{\epsilon T}+\int_{\epsilon T}^{(1-\epsilon) T}+\int_{(1-\epsilon) T}^{T} F\left(t, \omega_{1}, \omega_{2}\right) d t \geq \int_{\epsilon T}^{(1-\epsilon) T} F\left(t, \omega_{1}, \omega_{2}\right) d t .
$$

So

$$
\begin{aligned}
r \cdot \frac{\Psi\left(\omega_{1}, \omega_{2}\right)}{\Phi\left(\omega_{1}, \omega_{2}\right)} & =r \cdot \frac{\int_{0}^{T} F\left(t, \omega_{1}, \omega_{2}\right) d t}{\frac{1}{2}\left\|\omega_{1}\right\|_{\alpha}^{2}+\frac{1}{2}\left\|\omega_{2}\right\|_{\beta}^{2}-H\left(\omega_{1}, \omega_{2}\right)} \\
& \geq r \cdot \frac{\int_{\epsilon T}^{(1-\epsilon) T} F\left(t, \Gamma(2-\alpha) c_{1}, \Gamma(2-\beta) c_{2}\right) d t}{\rho \Delta_{2}\left(c_{1}^{2}+c_{2}^{2}\right)} \\
& =\frac{\kappa d}{2 M} \cdot \frac{\int_{\epsilon T}^{(1-\epsilon) T} F\left(t, \Gamma(2-\alpha) c_{1}, \Gamma(2-\beta) c_{2}\right) d t}{\rho \Delta_{2}\left(c_{1}^{2}+c_{2}^{2}\right)} \\
& >T \cdot \max _{t \in[0, T],(u, v) \in \Omega(d)} F(t, u, v) \\
& =T \cdot \max _{t \in[0, T],(u, v) \in \Omega\left(\frac{2 M r}{\kappa}\right)} F(t, u, v) \geq \sup _{\left.\left.(u, v) \in \Phi^{-1}(]-\infty, r\right]\right)} \Psi(u, v) .
\end{aligned}
$$

Thus we can fix $\rho$ such that

$$
\sup _{\left.\left.(u, v) \in \Phi^{-1}(]-\infty, r\right]\right)} \Psi(u, v)<\rho<r \frac{\Psi(u, v)}{\Phi(u, v)} .
$$


By Proposition 2.3 we have

$$
\sup _{\lambda \geq 0} \inf _{(u, v) \in X}(\Phi(u, v)+\lambda(\rho-\Psi(u, v)))<\inf _{(u, v) \in X} \sup _{\lambda \geq 0}(\Phi(u, v)+\lambda(\rho-\Psi(u, v))) .
$$

So, according to Theorem 2.2, for each interval $\Lambda \subset[0,+\infty)$ and $\varrho>0$ we have: for any $\lambda \in \Lambda$ and $G \in\left(G_{0}\right)$, there exists $\delta>0$ such that, for every $\mu \in[0, \delta], \Phi^{\prime}(u, v)-\lambda \Psi^{\prime}(u, v)-$ $\mu J^{\prime}(u, v)=0$ has at least three solutions in $X$ with norms less than $\varrho$. Therefore problem $\left(P_{\lambda, \mu}\right)$ has at least three solutions in $X$ with norms less than $\varrho$.

For the particular case of $F(t, u, v)=\varphi(t) f(u, v)$, where $\varphi(t) \in L^{1}([0, T] ; \mathbf{R}) \backslash\{0\}, f(u, v) \in$ $C^{1}\left(\mathbf{R}^{2}, \mathbf{R}\right)$, we can deduce the following two corollaries of Theorems 3.1 and 3.2, respectively.

Corollary 3.3 Assume that (H0) and (H1) hold. Moreover, assume that there exist a constant $\eta \geq 0$ and a constant vector $\bar{\omega}=\left(\omega_{1}, \omega_{2}\right) \in \mathbf{R}^{2} \backslash\{(0,0)\}$ such that

$(\mathrm{B} 1)^{\prime}$

$$
\max _{t \in[0, T]} \varphi(t) \cdot \max \left\{\limsup _{(u, v) \rightarrow 0} \frac{f(u, v)}{|u|^{2}+|v|^{2}}, \limsup _{|(u, v)| \rightarrow+\infty} \frac{f(u, v)}{|u|^{2}+|v|^{2}}\right\} \leq \eta
$$

$(\mathrm{B} 2)^{\prime}$

$$
\frac{2 T M \eta}{\kappa}<\frac{f\left(\omega_{1}, \omega_{2}\right) \int_{0}^{T} \varphi(t) d t}{\rho\left(\left\|\omega_{1}\right\|_{\alpha}^{2}+\left\|\omega_{2}\right\|_{\beta}^{2}\right)}
$$

Then, for each compact interval $\left[a_{1}, a_{2}\right] \subset\left(\lambda_{1}, \lambda_{2}\right)$, there exists a positive constant $\varrho$ with the following property: for every $\lambda \in\left[a_{1}, a_{2}\right]$ and for two Carathéodory functions $G_{u}, G_{v}$ satisfying (G0), there exists $\delta>0$ such that, for each $\mu \in[0, \delta)$, problem $\left(P_{\lambda, \mu}\right)$ has at least three weak solutions with norms less than $\varrho$.

Corollary 3.4 Assume that ( $\mathrm{H} 0)$ and $(\mathrm{H} 1)$ hold and there exist five positive constants $l_{0}$, $q_{0}, d, \theta_{1}, \theta_{2}$ and a constant vector $\bar{c}=\left(c_{1}, c_{2}\right) \in \mathbf{R}^{2}, c_{1}, c_{2}>0$, with $d<2 M \triangle_{1}\left(c_{1}^{2}+c_{2}^{2}\right)$ and $\theta_{1}, \theta_{2} \in[0,2)$, such that

(B3) $\varphi(t) f(u, v) \geq 0$ for all $t \in[0, \epsilon T] \cup[(1-\epsilon) T, T],|u| \leq \Gamma(2-\alpha) c_{1}$ and $|v| \leq \Gamma(2-$ $\beta) c_{2}$

(B4) $|f(u, v)| \leq l_{0}\left(|u|^{\theta_{1}}+|v|^{\theta_{2}}\right)+q_{0}$ for every $(u, v) \in X$;

$(\mathrm{B} 5)^{\prime}$

$$
\max _{(u, v) \in \Omega(d)} f(u, v)<\frac{d \kappa}{2 M\|\varphi\|_{L^{1}}} \cdot \frac{f\left(\Gamma(2-\alpha) c_{1}, \Gamma(2-\beta) c_{2}\right) \int_{\epsilon T}^{(1-\epsilon) T} \varphi(t) d t}{\rho \triangle_{2}\left(c_{1}^{2}+c_{2}^{2}\right)}
$$

where $\Omega(d)=\left\{(u, v) \in \mathbf{R}^{2}:|u|^{2}+|v|^{2} \leq d\right\}$.

Then there exist an open interval $\Lambda \subset[0,+\infty)$ and a positive real number $\varrho$ with the following property: for every $\lambda \in \Lambda$ and for two Carathéodory functions $G_{u}, G_{v}$ satisfying (G0), there exists $\delta>0$ such that, for each $\mu \in[0, \delta)$, problem $\left(P_{\lambda, \mu}\right)$ has at least three weak solutions with norms less than $\varrho$.

Finally, we present two examples to illustrate our abstract results. 
Example 3.5 Consider the following fractional boundary value problem:

$$
\left\{\begin{array}{l}
{ }_{t} D_{2}^{0.6}\left((1+t) \cdot{ }_{0} D_{t}^{0.6} u(t)\right)=\lambda F_{u}(t, u, v)+\mu G_{u}(t, u, v)+h_{1}(u), \quad 0<t<2, \\
{ }_{t} D_{2}^{0.8}\left(\frac{1}{3} \cdot{ }_{0} D_{t}^{0.8} v(t)\right)=\lambda F_{v}(t, u, v)+\mu G_{v}(t, u, v)+h_{2}(v), \quad 0<t<2, \\
u(0)=u(2)=0, \quad v(0)=v(2)=0,
\end{array}\right.
$$

where $T=2, \alpha=0.6, \beta=0.8, a(t)=1+t, b(t)=\frac{1}{3}$, and $h_{1}(u)=\frac{1}{20} u, h_{2}(v)=\frac{1}{30} \sin v$. Moreover, for all $(t, u, v) \in[0,2] \times \mathbf{R}^{2}$, put

$$
F(t, u, v)=10(1-2 t)\left(u^{2}+v^{2}\right)\left(\frac{1}{\ln \left(e+u^{2}+v^{2}\right)}-1\right)
$$

and

$$
G(t, u, v)=\left(1+t^{2}\right)\left(|u|^{\frac{5}{4}}+|v|^{\frac{4}{3}}\right) .
$$

Obviously, $h_{1}, h_{2}: \mathbf{R} \rightarrow \mathbf{R}$ are two Lipschitz continuous functions with the Lipschitz constants $L_{1}=\frac{1}{10}, L_{2}=\frac{1}{30}$ and $h_{1}(0)=h_{2}(0)=0 ; F(t, 0,0)=G(t, 0,0)=0$ for all $t \in[0,2]$, and conditions (F0), (G0), and (H0) hold. By simple calculations we get

$$
a_{0}=1, \quad b_{0}=\frac{1}{3}, \quad \rho \approx 1.3494, \quad \kappa \approx 0.6506, \quad M \approx 3.7271 .
$$

Taking $\eta=\frac{1}{60}$, we easily verify that (B1) is satisfied. Moreover, we have $\lambda_{1} \geq \frac{1}{229}$ and $\lambda_{2} \geq$ 2.6184. In fact,

$$
\begin{aligned}
\lambda_{1} & =\inf _{(u, v) \in X} \frac{\|u\|_{0.6}^{2}+\|v\|_{0.8}^{2}+2 H(u, v)}{20 \int_{0}^{2}(2 t-1) d t\left(u^{2}+v^{2}\right)\left(1-\frac{1}{\ln \left(e+u^{2}+v^{2}\right)}\right)} \\
& =\frac{1}{20 \int_{0}^{2}(2 t-1) d t} \cdot \inf _{(u, v) \in X} \frac{\|u\|_{0.6}^{2}+\|v\|_{0.8}^{2}+2 H(u, v)}{\left(u^{2}+v^{2}\right)\left(1-\frac{1}{\ln \left(e+u^{2}+v^{2}\right)}\right)} \\
& \geq \frac{1}{40} \cdot \inf _{(u, v) \in X} \frac{\kappa\left(\|u\|_{0.6}^{2}+\|v\|_{0.8}^{2}\right)}{M\left(\|u\|_{0.6}^{2}+\|v\|_{0.8}^{2}\right)\left(1-\frac{1}{\ln \left(e+u^{2}+v^{2}\right)}\right)} \\
& \geq \frac{1}{229}
\end{aligned}
$$

and $\lambda_{2} \geq \frac{\kappa}{2 T M \eta} \approx 2.6184$. On the other hand, choosing $\omega_{1}(t)=\Gamma(1.4) t(2-t)$ and $\omega_{2}(t)=$ $\Gamma(1.2) t(2-t)$, we have $\omega_{i}(0)=\omega_{i}(2)=0, i=1,2$, and

$$
{ }_{0} D_{t}^{0.6} \omega_{1}(t)=(2 t)^{0.4}-\frac{10}{7} t^{1.4}, \quad{ }_{0} D_{t}^{0.8} \omega_{2}(t)=t^{0.2}-\frac{5}{3} t^{1.2} .
$$

Hence we have $\left\|\omega_{1}\right\|_{0.6}^{2} \approx 3.8275,\left\|\omega_{2}\right\|_{0.8}^{2} \approx 1.0596$, and

$$
\frac{2 T M \eta}{\kappa} \approx 0.3819<\frac{\int_{0}^{2} F\left(t, \omega_{1}, \omega_{2}\right) d t}{\rho\left(\left\|\omega_{1}\right\|_{0.6}^{2}+\left\|\omega_{2}\right\|_{0.8}^{2}\right)} \approx 0.7032,
$$

which implies that condition (B2) holds. Hence, by Theorem 3.1, for any compact interval $\left[a_{1}, a_{2}\right] \subset\left(\frac{1}{229}, 2.6184\right)$, there exist a positive constant $\varrho$ with the following property: for 
every $\lambda \in\left[a_{1}, a_{2}\right]$, there exists $\delta>0$ such that, for each $\mu \in[0, \delta)$, problem (3.10) has at least three weak solutions with norms less than $\varrho$.

Example 3.6 Consider the following fractional differential system:

$$
\left\{\begin{array}{l}
{ }_{t} D_{1}^{0.75}\left((2+t) \cdot{ }_{0} D_{t}^{0.75} u(t)\right)=\lambda F_{u}(t, u, v)+\mu G_{u}(t, u, v)+h_{1}(u), \quad 0<t<1, \\
{ }_{t} D_{1}^{0.8}\left(\left(1+t^{3}\right) \cdot{ }_{0} D_{t}^{0.8} v(t)\right)=\lambda F_{v}(t, u, v)+\mu G_{v}(t, u, v)+h_{2}(v), \quad 0<t<1, \\
u(0)=u(1)=0, \quad v(0)=v(1)=0,
\end{array}\right.
$$

where $T=1, \alpha=0.75, \beta=0.8, a(t)=2+t, b(t)=1+t^{3}$, and $h_{1}(u)=\frac{1}{4} \sin u, h_{2}(v)=\frac{1}{18} v$. Moreover, for all $(t, u, v) \in[0,1] \times \mathbf{R}^{2}$, put $F(t, u, v)=\varphi(t)\left(|u|^{\frac{5}{4}}+|v|^{\frac{4}{3}}\right)$, where

$$
\varphi(t)= \begin{cases}\frac{1}{4}-t, & t \in\left[0, \frac{3}{8}\right] \\ -\frac{1}{2}+t, & t \in\left[\frac{3}{8}, 1\right]\end{cases}
$$

and $G(t, u, v)=t^{2}\left(|u|^{\frac{3}{2}}+|v|^{\frac{6}{5}}\right)$.

Obviously, $h_{1}, h_{2}: \mathbf{R} \rightarrow \mathbf{R}$ are two Lipschitz continuous functions with the Lipschitz constants $L_{1}=\frac{1}{4}, L_{2}=\frac{1}{9}$ and $h_{1}(0)=h_{2}(0)=0 ; F(t, 0,0)=G(t, 0,0)=0$ for all $t \in[0,1]$. By simple calculations we have that $a_{0}=2, b_{0}=1$, and

$$
M \approx 1.2302, \quad \kappa \approx 0.8520, \quad \rho \approx 1.1480 .
$$

Letting $\epsilon=\frac{1}{4}$, we obtain $P(\alpha, \epsilon) \approx 7.9576$ and $Q(\beta, \epsilon) \approx 4.4641$. Hence $\Delta_{1}=4.4641$ and $\Delta_{2}=7.9576$. Take $d=\frac{1}{2}, c_{1}=c_{2}=\frac{1}{6}, l_{0}=1, q_{0}>0, \theta_{1}=\frac{5}{4}$, and $\theta_{2}=\frac{4}{3}$. Then all the conditions in Corollary 3.4 are satisfied. In fact, conditions (B3) ${ }^{\prime}$ and (B4) $)^{\prime}$ hold, and by direct computation we have

$$
\frac{1}{2}=d<2 M \triangle_{1}\left(c_{1}^{2}+c_{2}^{2}\right) \approx 0.6102
$$

and

$$
\max _{(u, v) \in \Omega\left(\frac{1}{2}\right)} f(u, v) \approx 0.8147<\frac{8 d \kappa}{M\|\varphi\|_{L^{1}}} \cdot \frac{f(\Gamma(0.21), \Gamma(0.2)) \int_{\frac{1}{4}}^{\frac{3}{4}} \varphi(t) d t}{\rho \triangle_{2}} \approx 1.8223,
$$

which implies that condition (B3)' holds. By Corollary 3.4 there exist an open interval $\Lambda \subset[0,+\infty)$ and a positive constant $\varrho$ with the following property: for every $\lambda \in \Lambda$, there exists $\delta>0$ such that, for each $\mu \in[0, \delta)$, problem (3.11) has at least three weak solutions with norms less than $\varrho$.

\section{Conclusion}

In this paper, we investigated the existence of solutions for a class of fractional differential system with two control parameters. By constructing a variational framework and using some critical points in theorems of Ricceri, we obtained several new existence results for at least three weak solutions in terms of different values of the two parameters $\lambda, \mu$. It is worth remarking that we suppose the primitive function $G$ of $G_{u}, G_{v}$ to satisfy a general 
growth condition allowing us to apply a variational method. In addition, we obtain the multiplicity results for two cases: where the primitive function $F$ of $F_{u}, F_{v}$ is asymptotically quadratic and where it is subquadratic as $|(u, v)| \rightarrow \infty$.

\section{Acknowledgements}

The authors would like to express their deep thanks to the referee for valuable suggestions for the revision and improvement of the manuscript.

Funding

The research is supported by Hunan Provincial Natural Science Foundation of China (2019JJ40068).

\section{Abbreviations}

Not applicable.

\section{Availability of data and materials}

Not applicable.

\section{Competing interests}

The authors declare that they have no competing interests.

\section{Authors' contributions}

The authors declare that the study was realized in collaboration with the same responsibility. All authors read and approved the final manuscript.

\section{Publisher's Note}

Springer Nature remains neutral with regard to jurisdictional claims in published maps and institutional affiliations.

Received: 12 October 2018 Accepted: 1 April 2019 Published online: 16 April 2019

\section{References}

1. Diethelm, K.: The Analysis of Fractional Differential Equation. Springer, Heidelberg (2010)

2. Kilbas, A.A., Srivastava, H.M., Trujillo, J.J.: Theory and Applications of Fractional Differential Equations. Elsevier, Amsterdam (2006)

3. Podlubny, I.: Fractional Differential Equations. Mathematics in Science and Engineering, vol. 198. Academic Press, New York (1999)

4. Zhou, Y.: Basic Theory of Fractional Differential Equations. World Scientific, Singapore (2014)

5. Xu, X., Jiang, D., Yuan, C.: Multiple positive solutions for the boundary value problem of a nonlinear fractional differential equation. Nonlinear Anal. 71, 4676-4688 (2009)

6. Liang, S., Zhang, J.: Positive solutions for boundary value problems of nonlinear fractional equation. Nonlinear Anal. 71, 5545-5550 (2009)

7. Wang, J., Xiang, H.: Upper and lower solutions methods for a class of singular fractional boundary value problems with p-Laplacian operator. Abstr. Appl. Anal. 2010, Article ID 971824 (2010)

8. Jia, M., Liu, X.: Multiplicity of solutions for integral boundary value problems of fractional differential equations with upper and lower solutions. Appl. Math. Comput. 232, 313-323 (2014)

9. Ahmad, B., Sivasundaram, S.: On four-point nonlocal boundary value problems of nonlinear integro-differential equations of fractional order. Appl. Math. Comput. 217, 480-487 (2010)

10. Zhao, Y., Chen, H., Huang, L.: Existence of positive solutions for nonlinear fractional functional differential equation. Comput. Math. Appl. 64, 3456-3467 (2012)

11. Fečkan, M., Zhou, Y., Wang, J.: On the concept and existence of solution for impulsive fractional differential equations. Commun. Nonlinear Sci. Numer. Simul. 17, 3050-3060 (2012)

12. Agarwal, R.P., Benchohra, M., Hamani, S.: A survey on existence results for boundary value problems of nonlinear fractional differential equations and inclusions. Acta Appl. Math. 109, 973-1033 (2010)

13. Ahmad, B., Alsaedi, A.: Existence and uniqueness of solutions for coupled systems of higher-order nonlinear fraction differential equations. Fixed Point Theory Appl. 2010, Article ID 364560 (2010)

14. Rodríguez-López, R., Tersian, S.: Multiple solutions to boundary value problem for impulsive fractional differential equations. Fract. Calc. Appl. Anal. 17(4), 1016-1038 (2014)

15. Bai, C.: Infinitely many solutions for a perturbed nonlinear fractional boundary-value problem. Electron. J. Differ. Equ. 2013, $136(2013)$

16. Zhang, X., Liu, L., Wu, Y.: Variational structure and multiple solutions for a fractional advection-dispersion equation. Comput. Math. Appl. 68, 1794-1805 (2014)

17. Cabada, A., Hamdi, Z.: Nonlinear fractional differential equations with integral boundary value conditions. Appl. Math Comput. 228, 251-257 (2014)

18. Bai, C., Fang, J.: The existence of a positive solution for a singular coupled system of nonlinear fractional differential equations. Appl. Math. Comput. 150, 611-621 (2004)

19. Henderson, J., Luca, R.: Positive solutions for a system of nonlocal fractional boundary value problems. Fract. Calc. Appl. Anal. 16(4), 985-1008 (2013)

20. Jiao, F., Zhou, Y.: Existence of solutions for a class of fractional boundary value problems via critical point theory. Comput. Math. Appl. 62, 1181-1199 (2011) 
21. Jiao, F., Zhou, Y.: Existence results for fractional boundary value problem via critical point theory. Int. J. Bifurc. Chaos $22,1250086(2012)$

22. Sun, H., Zhang, Q: Existence of solutions for a fractional boundary value problem via the mountain pass method and an iterative technique. Comput. Math. Appl. 64, 3436-3443 (2012)

23. Klimek, M., Odzijewicz, T., Malinowska, A.B.: Variational methods for the fractional Sturm-Liouville problem. J. Math. Anal. Appl. 416, 402-426 (2014)

24. Zhao, Y., Chen, $\mathrm{H}$., Xu, C.: Nontrivial solutions for impulsive fractional differential equations via Morse theory. Appl. Math. Comput. 307, 170-179 (2017)

25. Heidarkhani, S., Zhao, Y., Caristi, G., Afrouz, G.A., Moradi, S.: Infinitely many solutions for perturbed impulsive fractional differential systems. Appl. Anal. 96(8), 1401-1424 (2017)

26. Galewski, M., Molica Bisci, G.: Existence results for one-dimensional fractional equations. Math. Methods Appl. Sci. 39, 1480-1492 (2016)

27. Nyamoradi, N., Rodríguez-López, R.: Multiplicity of solutions to fractional Hamiltonian systems with impulsive effects. Chaos Solitons Fractals 102, 254-263 (2017)

28. Zhao, Y., Shi, X., Chen, H.: Multiplicity results for a class of fractional differential equations with impulse. Adv. Differ. Equ. 2018, 341 (2018)

29. Ahmad, B., Nieto, J.J.: Existence results for a coupled system of nonlinear fractional differential equations with three-point boundary conditions. Comput. Math. Appl. 58, 1838-1843 (2009)

30. Sun, S., Li, Q., Li, Y.: Existence and uniqueness of solutions for a coupled system of multi-term nonlinear fractional differential equations. Comput. Math. Appl. 64, 3310-3320 (2012)

31. Zhao, Y., Chen, H., Qin, B.: Multiple solutions for a coupled system of nonlinear fractional differential equations via variational methods. Appl. Math. Comput. 257, 417-427 (2015)

32. Zhao, Y., Tang, L.: Multiplicity results for impulsive fractional differential equations with $p$-Laplacian via variational methods. Bound. Value Probl. 2017, Article ID 123 (2017)

33. Bonanno, G., Molica Bisci, G.: Infinitely many solutions for a boundary value problems with discontinuous nonlinearities. Bound. Value Probl. 2009, Article ID 670675 (2009)

34. Bonanno, G., Marano, S.A.: On the structure of the critical set of non-differentiable functions with a weak compactness condition. Appl. Anal. 89, 1-10 (2010)

35. Vinagre, B.M., Monje, C., Calderon, A.: Fractional order systems and fractional order control actions. In: IEEE Conference on Decision and Control, Las Vegas, NV, USA, pp. 2550-2554 (2002)

36. Poinot, T., Trigeassou, J.C.: Identification of fractional systems using an output-error technique. Nonlinear Dyn. 38 133-154 (2004)

37. Qian, D., Li, C., Agarwal, R.P., Wong, P.J.Y.: Stability analysis of fractional differential system with Riemann-Liouville derivative. Math. Comput. Model. 52, 862-874 (2010)

38. Atanackovic, T.M., Stankovic, B:: On a system of differential equations with fractional derivatives arising in rod theory. J. Phys. A 37, 1241-1250 (2004)

39. Ricceri, B.: A further three critical points theorem. Nonlinear Anal. TMA 71, 4151-4157 (2009)

40. Ricceri, B.: A three critical points theorem revisited. Nonlinear Anal. TMA 70, 3084-3089 (2009)

41. Ricceri, B.: Existence of three solutions for a class of elliptic eigenvalue problem. Math. Comput. Model. 32, 1485-1494 (2000)

42. Zeidler, E.: Nonlinear Functional Analysis and Applications, vol. 2. Springer, Berlin (1990)

\section{Submit your manuscript to a SpringerOpen ${ }^{\circ}$ journal and benefit from:}

- Convenient online submission

- Rigorous peer review

- Open access: articles freely available online

- High visibility within the field

- Retaining the copyright to your article

Submit your next manuscript at $\gg$ springeropen.com 